\section{Co-Infection with 4 Species of Mycobacteria Identified by Using Next-Generation Sequencing}

\author{
Lulan Wang, ${ }^{1}$ Dakai Liu, ${ }^{1}$ Lok Yung, \\ George David Rodriguez, Nishant Prasad, \\ Sorana Segal-Maurer, Vishnu Singh, \\ Ellee Vikram, Angela Zou, Genhong Cheng, \\ William Harry Rodgers
}

Author affiliations: University of California, Los Angeles, California, USA (L. Wang, E. Vikram, A. Zou, G. Cheng); New York-Presbyterian Queens Hospital, Flushing, New York, USA (D. Liu, L. Yung, G.D. Rodriguez, N. Prasad, S. Segal-Maurer, V. Singh, W.H. Rodgers); Weil Cornell Medical College, New York (W.H. Rodgers)

DOI: https://doi.org/10.3201/eid2711.203458

We identified co-infection with 4 species of mycobacteria in a woman in New York, New York, USA, by using nextgeneration sequencing. This procedure is useful for identifying co-infections with multiple mycobacteria, tracing the geographic origin of strains, investigating transmission dynamics in susceptible populations, and gaining insight into prevention and control.

$\mathrm{M}$ ycobacteria are major human pathogens; $\approx 13$ million persons in the United States live with Mycobacterium tuberculosis complex (MTBC) infection, and incidence of nontuberculous mycobacterial (NTM) pulmonary disease is increasing worldwide. The challenges of managing MTBC and M. avium complex (MAC) co-infection are well described, including the risk for falsely interpreted Xpert RIF (rifampin) results $(1,2)$. MTBC and M. abscessus co-infection has been described in case reports only $(3,4)$. We describe co-infection with 4 species of mycobacteria.

In July 2019, an 82-year-old Asian woman was hospitalized in Flushing, New York, USA, for persistent fever associated with worsening weakness. Computed tomography of her chest showed near-complete atelectasis of the left upper lobe, hyperinflation in other areas, and a small left-sided pleural effusion. Scattered nodular opacities in a tree-in-bud pattern and pulmonary granulomas were present throughout the lungs, and discontinuity of the left upper lobe bronchus was noted. Cultures of blood, urine, stool, and respiratory specimens yielded negative results for nonmycobacteria.

In a sputum sample collected for routine mycobacterial testing, fluorochrome staining exhibited

${ }^{1}$ These first authors contributed equally to this article. rare acid-fast bacilli, and $\mathrm{MTBC}$ was detected by using Xpert MTB/RIF (Cepheid, https://www.cepheid.com). We then inoculated a Lowenstein-Jensen Gruft slant with sputum, incubated it at $37^{\circ} \mathrm{C}$, and inoculated VersaTREK Myco bottles containing Modified Middlebrook 7H9 Broth with Sponges (Thermo Fisher, https://www.thermofisher.com) and incubated them at $35^{\circ} \mathrm{C}$. No isolate was recovered from the Lowenstein-Jensen Gruft slant. Only MAC was detected by AccuProbe (Hologic, https://www. hologic.com) in Kinyoun-positive culture from the Myco bottles. One week later, another sputum sample with Kinyoun-positive growth from the Myco bottles was negative for MAC, MTBC, M. gordonae, and M. kansasii. M. abscessus was identified on the Lowenstein-Jensen Gruft slant.

Considering the sensitivity limit and narrow species coverage of AccuProbe and the difficulty of identifying mycobacteria by culturing and because of growth interference among different mycobacteria, we conducted next-generation sequencing (NGS) by using Hiseq3000 (Illumina, https:/ / www. illumina.com) on the supernatant of the first sputum culture. NGS yielded $\approx 175$ million reads, each with a quality score of $>35$. We checked NGS data for quality control by using FastQC (Galaxy, https:// usegalaxy.org). All steps and programs used the data processing pipeline from Galaxy, an opensource, web-based platform for data-intensive biomedical research. Each read identified had a quality control score of 39.4 and an average guanine-cytosine content of $68 \%$. Only $0.69 \%$ of bases resulted in no hits and were not identifiable. We performed De Novo classification by using De Novo Assembly Unicycler, Quast QC, and Kraken Classification (Galaxy) and generated coverage and depth data by using BWA Aligner and SAMtools Depth (Galaxy). We aligned the reads, visualized onto bacteria reference genomes by using Bowtie2 (Galaxy) and converted into BED (Browser Extensible Data) files followed by Bedtools Merge, Multicov (https:// bedtools.readthedocs.io).

The genome visualization pipeline confirmed 4 genomic traces of Mycobacterium strains (Figure): M. yongonense strain 05-1390 (GenBank accession no. NC_021715.1), M. tuberculosis strain FDAARGOS_757 (GenBank accession no. CP054013.1), Mycobacterium sp. MOTT36Y (GenBank accession no. NC_017904.1), and M. abscessus ATCC 19977 (GenBank accession no. CU458896.1). M. yongonense was identified with a genome coverage of $88.73 \%$ (4.9 $\mathrm{Mb}$ mapped of 5.5-Mb genome) and a read depth of $1,224 \times$. M. tuberculosis was identified with a genome 
A

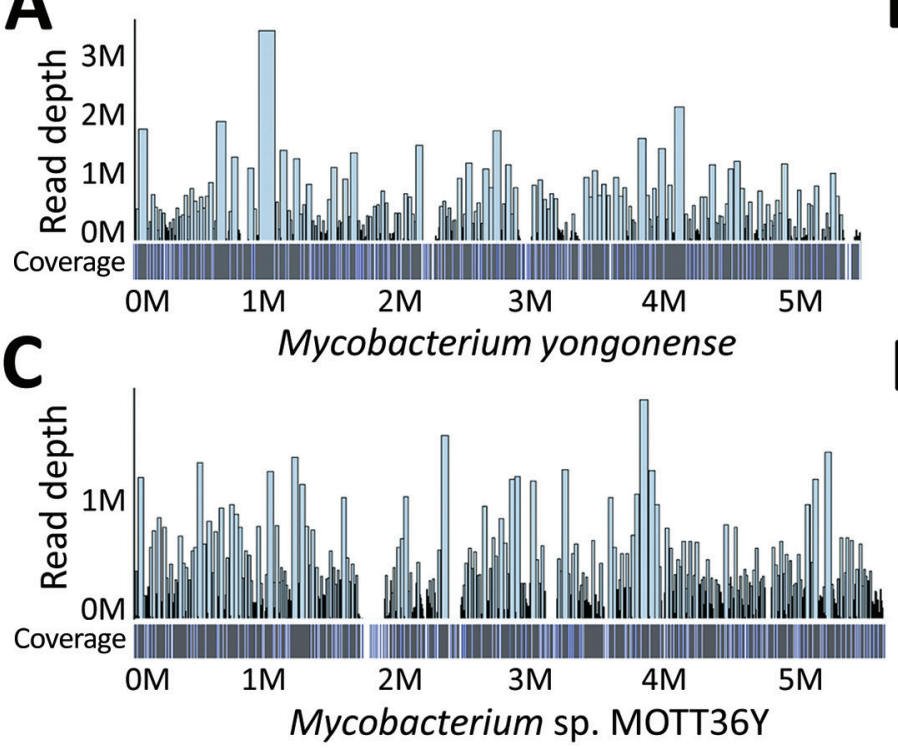

B
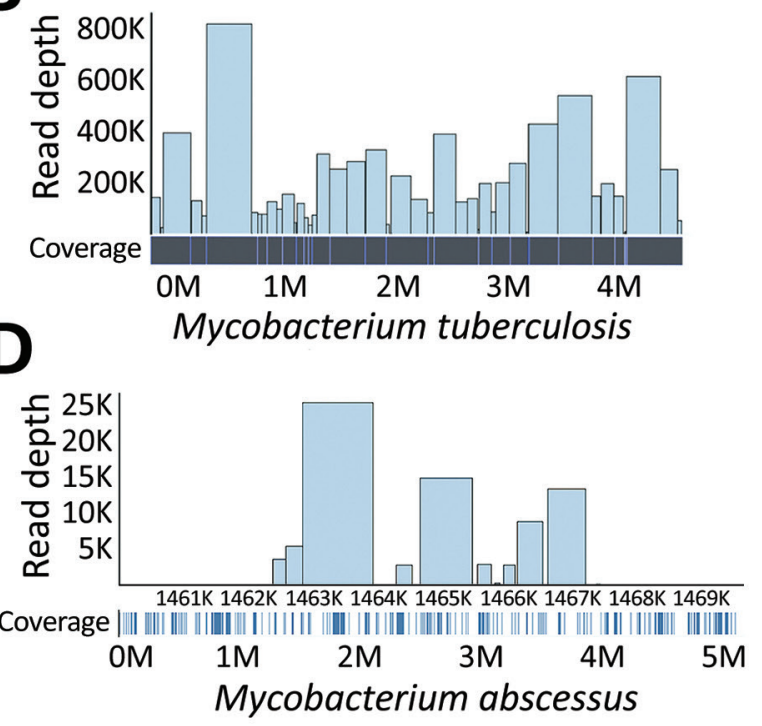

Figure. Genomic coverage and depth map of 4 Mycobacterium strains identified by using next-generation sequencing on isolates from a woman in New York, New York, USA. The reads were aligned by using bacteria reference genomes with Bowtie 2 and visualized by using aligned BED file (https://bedtools.readthedocs.io). A) M. yongonense; B) M. tuberculosis; C) Mycobacterium sp. MOTT36Y; D) M. abscessus.

coverage of $99.99 \%$ (4.4 $\mathrm{Mb}$ mapped of $4.4-\mathrm{Mb}$ genome) and a read depth of $63 \times$. Mycobacterium sp. was identified with a genome coverage of $94.41 \%$ (5.3 $\mathrm{Mb}$ mapped of 5.6-Mb genome) and a read depth of $1210 \times$. M. abscessus was identified with a genome coverage of only $2.75 \%(0.14 \mathrm{Mb}$ mapped of $5.1-\mathrm{Mb}$ genome) and a read depth of $8 \times$ (Table). The mycobacteria identified by NGS were verified by various mycobacteria tests.

We obtained the consensus sequence for 4 strains of bacteria by using MEGAHIT (Galaxy) and generated a BLAST (https://blast.ncbi.nlm.nih.gov/Blast. cgi) tree based on minimum evolution at the species level by using $\geq 15 \mathrm{kbp}$ from each sequence. Assembly on MTBC sequencing data yielded a total consensus sequence of 4,376,826 bp and 78,208 single-nucleotide polymorphism sites (1.79\%). Analysis by BLAST and Mykrobe (https:/ / www.mykrobe.com) revealed that the MTBC isolate belongs to sublineage 2.2.

The patient received RIPE therapy (rifampin, isoniazid, pyrazinamide, and ethambutol), along with amikacin, tigecycline, and azithromycin. At 6 months, RIPE therapy was completed. At 9 months, sputum culture was negative. The patient continues to take amikacin, tigecycline, and azithromycin as an outpatient with close follow-up.

Identification of co-infection with mycobacteria is necessary for diagnosis and treatment (5). Treatment regimens and duration remain species specific because of unique resistance mechanisms. To achieve the greatest potential for success while minimizing toxicities, early empiric treatment should account for clinical characteristics of MTBC and NTM co-infection and strain identification.

Our report highlights the value of NGS for identifying multiple mycobacteria co-infections in populations with high susceptibility to and prevalence of MTB and NTM (i.e., immigrants, immunocompromised patients, and international travelers). NGS can trace the geographic origin of the $\mathrm{Myco}$ bacterium strain. These features, in combination with a patient's epidemiologic exposure and travel history, could elucidate the potential time and location of infection acquisition. NGS could also be used to identify drug-resistance genes to guide targeted therapy.

$\begin{aligned} & \text { Table. Classification and coverage of multiple Mycobacterium strains identified by using next-generation sequencing of isolates from a } \\
& \text { woman in New York, New York, USA }\end{aligned}$
\begin{tabular}{lcccc} 
& \\
\hline Strain & Genome size, bp & Coverage, bp & Coverage, \% & Read depth \\
\hline Mycobacterium avium complex sp YG & $5,521,023$ & $4,900,000$ & 88.752 & $1223.91 \times$ \\
M. tuberculosis complex & $4,405,981$ & $4,405,474$ & 99.999 & 39.4 \\
Mycobacterium sp. Mott36Y & $5,613,626$ & $5,300,000$ & 94.413 & $1209.32 \times$ \\
M. abscessus & $5,090,491$ & 139,997 & 2.750 & 39.4 \\
\hline
\end{tabular}




\section{Acknowledgments}

We thank Jun Young Choi, Debra Harragan Jokisch, Eric Konadu, Marian Memmer, Joseph Pilz, Huimin Wu for their technical assistance and Carl Urban for manuscript review.

The sequenced isolate in this manuscript was submitted to GenBank (accession no. CP074075). Details of submission are SUBID SUB9558401; BioProject PRJNA726345: BioSample SAMN18928235; Organism Mycobacterium tuberculosis 2.2.

\section{About the Authors}

Dr. Wang is a postdoctoral fellow working in the Department of Microbiology, Immunology and Molecular Genetics, University of California, Los Angeles. His research focuses on immunology, virology, and molecular diagnostics. Dr. Liu is the laboratory director for Microbiology, Immunology and Molecular Diagnostics, Department of Pathology and Clinical Laboratories, New York-Presbyterian Queens Hospital. His research focus is developing rapid molecular assays for pathogen diagnosis.

\section{References}

1. Bazzi AM, Abulhamayel Y, Rabaan AA, Al-Tawfiq JA. The impact of the coexistence of Mycobacterium avium with Mycobacterium tuberculosis on the result of GeneXpert and MGIT susceptibility test. J Infect Public Health. 2020;13:8279. PubMed https://doi.org/10.1016/j.jiph.2020.01.006

2. Sarro YDS, Kone B, Diarra B, Kumar A, Kodio O, Fofana DB, et al. Simultaneous diagnosis of tuberculous and non-tuberculous mycobacterial diseases: time for a better patient management. Clin Microbiol Infect Dis. 2018;3:1-8. https:// doi.org/10.15761/CMID.1000144

3. Sohn S, Wang S, Shi H, Park S, Lee S, Park KT. Mixed infection of Mycobacterium abscessus subsp. abscessus and Mycobacterium tuberculosis in the lung. Korean J Thorac Cardiovasc Surg. 2017;50:50-3. https://doi.org/10.5090/ kjtcs.2017.50.1.50

4. Ishiekwene C, Subran M, Ghitan M, Kuhn-Basti M, Chapnick E, Lin YS. Case report on pulmonary disease due to coinfection of Mycobacterium tuberculosis and Mycobacterium abscessus: difficulty in diagnosis. Respir Med Case Rep. 2017;20:123-4. https://doi.org/10.1016/j.rmcr.2017.01.011

5. Feng Z, Bai X, Wang T, Garcia C, Bai A, Li L, et al. Differential responses by human macrophages to infection with Mycobacterium tuberculosis and non-tuberculous mycobacteria. Front Microbiol. 2020;11:116. https://doi. org/10.3389/fmicb.2020.00116

Address for correspondence: Dakai Liu and William H. Rodgers, Department of Pathology and Clinical Laboratories, New York Presbyterian Queens Hospital, 56-45 Main St., Flushing, NY 11355, USA; email: da19165@nyp.org and whr9001@nyp.org; Genhong Cheng, Department of Microbiology, Immunology \& Molecular Genetics, University of California, Los Angeles, 615 Charles Young Dr S, Los Angeles, CA 90095, USA; email: gcheng@mednet.ucla.edu

\section{Fatal Co-infections with SARS-CoV-2 and Legionella pneumophila, England}

\author{
Victoria J. Chalker, Hugh Adler, Robert Ball, \\ Falguni Naik, Jessica Day, Baharak Afshar, \\ Amit K. Amin
}

Author affiliations: United Kingdom Health Security Agency, London, UK (V.J. Chalker, F. Naik, B. Afshar); St. Helen's \& Knowsley Teaching Hospitals National Health Service Trust, St. Helen's, UK (H. Adler, R. Ball); London Northwest University Healthcare National Health Service Trust, London (A.K. Amin)

DOI: https://doi.org/10.3201/eid2711.4121

Both Legionella pneumophila and severe acute respiratory syndrome coronavirus 2 (SARS-CoV-2) can cause pneumonia. L. pneumophila is acquired from water sources, sometimes in healthcare settings. We report 2 fatal cases of $L$. pneumophila and SARS-CoV-2 co-infection in England. Clinicians should be aware of possible L. pneumophila infections among SARS-CoV-2 patients.

工 egionnaires' disease, caused by Legionella bacteria, is a factor in community and healthcare acquired pneumonia. Legionella infection occurs from manmade water sources, including water aerosolized from cooling towers, spa pools, and water features, and from plumbing in hotels, workplaces, and healthcare facilities (1), where patients can be more susceptible to infection (1).

Severe acute respiratory syndrome coronavirus 2 (SARS-CoV-2) causes coronavirus disease (COVID-19), which also can cause pneumonia. Clinically differentiating Legionnaires' disease from COVID-19 requires laboratory diagnostics, such as urine antigen testing, PCR, and culture. The clinical focus on SARS-CoV-2 potentially causes underdiagnosis of L. pneumophila because clinicians might not suspect or investigate the bacterium, but co-infections have been reported. Documented co-infections in COVID-19 patients include human metapneumovirus (2), influenza (3), Chlamydia pneumoniae, Mycoplasma pneumoniae, non-COVID-19 coronavirus, enterovirus, rhinovirus, parainfluenza, and respiratory syncytial virus (4), and L. pneumophila in a case associated with a cruise ship (5). Rapid identification of co-infections is essential for managing and treating severe COVID-19 cases (6). We describe 2 cases of SARS-CoV-2 and L. pneumophila co-infection in patients admitted to hospitals in the United Kingdom.

In February 2020, a female patient $>65$ years of age was admitted in Addisonian crisis. She was dis- 